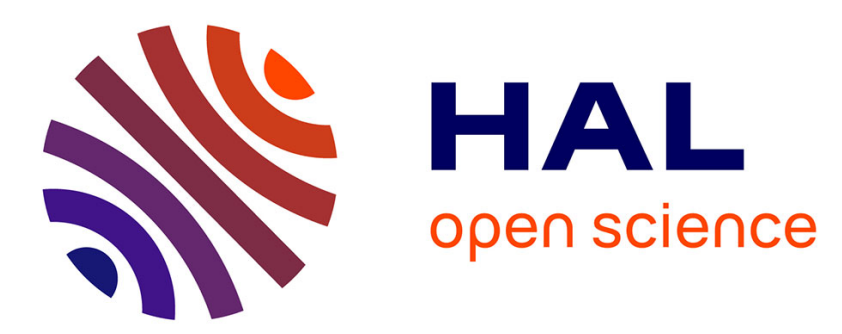

\title{
Dual microelectrodes decorated with nanotip arrays: Fabrication, characterization and spectroelectrochemical sensing
}

Thibault Bombail, Patrick Garrigue, Bertrand Goudeau, David Talaga, Laurent Bouffier, Sébastien Bonhommeau, Laurent Servant, Dodzi Zigah, Stéphane Arbault, Neso Sojic

\section{To cite this version:}

Thibault Bombail, Patrick Garrigue, Bertrand Goudeau, David Talaga, Laurent Bouffier, et al.. Dual microelectrodes decorated with nanotip arrays: Fabrication, characterization and spectroelectrochemical sensing. Electrochimica Acta, 2019, 328, pp.135105. 10.1016/j.electacta.2019.135105 . hal02374935

\author{
HAL Id: hal-02374935 \\ https://hal.science/hal-02374935
}

Submitted on 17 Dec 2020

HAL is a multi-disciplinary open access archive for the deposit and dissemination of scientific research documents, whether they are published or not. The documents may come from teaching and research institutions in France or abroad, or from public or private research centers.
L'archive ouverte pluridisciplinaire HAL, est destinée au dépôt et à la diffusion de documents scientifiques de niveau recherche, publiés ou non, émanant des établissements d'enseignement et de recherche français ou étrangers, des laboratoires publics ou privés. 


\title{
Dual microelectrodes decorated with nanotip arrays: Fabrication, characterization and spectroelectrochemical sensing
}

\author{
Thibault Bombail, Patrick Garrigue, Bertrand Goudeau, David Talaga, Laurent Bouffier, \\ Sébastien Bonhommeau, Laurent Servant, Dodzi Zigah, Stéphane Arbault**, Neso Sojic*
}

Univ. Bordeaux, CNRS, Bordeaux INP, ISM, UMR5255, 33400, Talence, France

\section{A R T ICLE INFO}

Article history:

Received 16 September 2019

Received in revised form 15 October 2019

Accepted 16 October 2019

Available online $\mathrm{xxx}$

\section{Keywords}

Microelectrode

Optical fiber

Spectroelectrochemistry

Scanning electrochemical microscopy

Surface-enhanced Raman sensing

\begin{abstract}
A B S T R A C T
Gold microelectrodes decorated with nanotips were developed for spectroelectrochemical experiments. These new dual probes were fabricated by combining fabrication processes of scanning near-field optical microscopy with photolithography. A nanotip array was produced at the surface of a coherent optical fiber bundle by a wet chemical etching step. The resulting nanostructured surface was sputter-coated with a thin gold layer. This gold film conferred plasmonic properties to the sharp nanotips and served as well as the electrode material to enable electrochemical reactions. A photolithographic process was used to define on the bundle surface nanotips-decorated microelectrodes with tunable dimensions (radii ranging between $20 \mu \mathrm{m}$ and $3.5 \mu \mathrm{m}$ ) individually or in an array format. The resulting microelectrodes with a regular nanotip pattern were characterized first by cyclic voltammetry. Numerical simulation was used to assess the electrochemical properties of these platforms and the influences of the recessed geometry and of the nanotips. Approach curves were recorded in negative and positive feedback modes of scanning electrochemical microscopy (SECM) on insulating and conducting substrates. Finally, spatially resolved Raman imaging allowed us to detect a mercaptobenzoic acid monolayer adsorbed on the microelectrode surface, demonstrating a surface-enhanced Raman scattering (SERS) effect induced by the gold-coated nanotips with a typical enhancement factor of $\sim 7 \times 10^{4}$. Such an approach introduces a reproducible method to fabricate promising SERS-active platforms with microelectrode behavior for SECM experiments.
\end{abstract}

\section{Introduction}

Scanning ElectroChemical Microscopy (SECM) is a powerful in situ technique to map the electrochemical or (bio)-chemical reactivity on various surfaces of complex samples, interfaces or materials [1]. Typically, the experiments are performed with a disk microelectrode, which is scanned in position and distance over the surface of the investigated sample. The disk microelectrode is usually fabricated by insulating a metal wire with glass or an epoxy resin. The fabrication steps ensure a precise disk geometry at the tip, which provides well-defined diffusional conditions for the dissolved redox species in the classic SECM configuration. However, different electrode geometries such as cones $[2,3]$, spheres [3-5], bands [6,7], rings [8], needles [9] or recessed disks [10] can be exploited with the support of numerical simulations to treat the corresponding faradaic responses and build up the

\footnotetext{
* Corresponding author.

** Corresponding author.

E-mail addresses: stephane.arbault@u-bordeaux.fr (S. Arbault); Neso.Sojic@enscbp.fr (N. Sojic)
}

approach curves toward the scanned surface. SECM has shown to be a very versatile technique pushing its limits towards new horizons: mapping the reactivity of various samples with better resolution, or with highly parallelized probes, or coupling the local electrochemical information with additional topographic and spectroscopic measurements [11-17]. This latter strategy has been pursued by several groups in recent years [18-22]. Indeed, the cross-correlation of spectroscopic information with local electrochemical measurements provides a powerful basis for increased selectivity and sensitivity. The ability to merge intimately such techniques that are based on different physical principles offers the opportunity to acquire various and complementary information on complex micro-environments. For example, SECM has been associated with a variety of optical/spectroscopic techniques: optical microscopy $[18,23]$, single-molecule fluorescence spectroscopy [24], infrared spectroscopy $[21,22]$ and with surface plasmon resonance $[25,26]$. SECM has also been successfully combined with Raman spectrometry [27-29]. More recently, SECM measurements with shear-force regulation of the probe position have been reported under a confocal Raman microspectrometer in order to study local corrosion phenomena [20]. Complementary information concerning the surface 
reactivity, topology, and chemical composition was collected on the same region of interest.

Raman spectroscopy is of particular interest because it provides a direct identification of molecular fingerprints of the sample without any labeling procedure or sample preparation. However, the main drawback of Raman measurements is the poor Raman scattering cross-section, which limits the sensitivity of the method. The inherently weak Raman signal can however be enhanced when the molecule is adsorbed onto a rough metallic surface and irradiated by a laser at an appropriate wavelength [30-32]. Such an approach is quoted as Surface-Enhanced Raman Scattering (SERS) spectroscopy [33,34]. A further increase can be obtained by working in either resonance or pre-resonance conditions. It means that the excitation wavelength should be tuned near an electronic absorption band of the molecular species. The same enhancement mechanisms operate at the Au or Ag nanotips in TERS (tip-enhanced Raman spectroscopy) where the magnetic field is strongly confined to the apex. The combination of TERS with SECM has been recently proposed for structural interrogation of electrochemically-generated intermediates $[35,36]$. A wide variety of approaches has been investigated for the fabrication of efficient plasmonic and SERS substrates, based among others, on electrochemical roughening and nanosphere lithography [37-40]. Advanced lithographic techniques such as electron-beam lithography and focused ion beam milling have also been used to fabricate more rationally designed nanostructures. An alternative strategy is to specifically engineer optical fiber bundles on their distal faces and use them as SERS substrates [41-49]. Various regular patterns such as microwells, nanotips, and nanotips surrounding microwells have been fabricated on the distal face (the proximal face being used for light injection/collection), depending on the wet-etching conditions and on the core and cladding doping materials [41,50-54].

Herein, we report the development of a dual spectroelectrochemical platform that involves embedded plasmonic structures with microelectrode capabilities. It is based on an array of sharp nanotips that were formed by wet chemical etching at the surface of an optical fiber bundle (Fig. 1). Such nanotip arrays have been applied previously for electrochemiluminescence imaging [55-58], for electrochemical surface patterning in SECM mode [13] and for shear-force positioning [12]. In the present work, the surface of the etched bundle was sputter-coated with a thin gold layer, which converts it into an effective plasmonic surface as well as into an electroactive nanotips-decorated microelectrode. The photolithographic process allows fabricating unprecedented recessed nanotip electrodes with disk shape and tunable micrometric dimensions. The electrochemical responses of the resulting microelectrodes with a regular nanotip pattern were characterized by cyclic voltammetry. Then, SECM approach curves were recorded on insulating and conducting substrates. The electrochemical results were
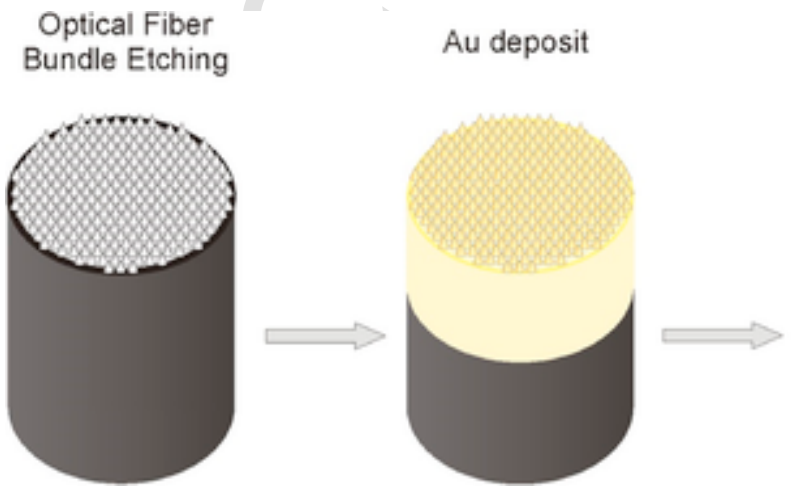
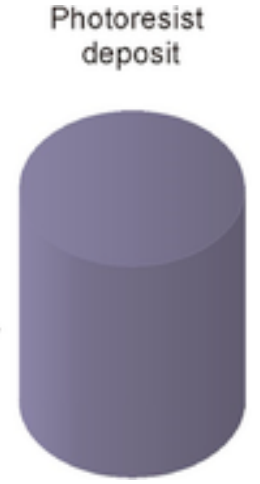

confronted to numerical simulations. Finally, spatially resolved Raman experiments have been performed to detect a mercaptobenzoic acid monolayer adsorbed on the microelectrode surface, demonstrating a SERS effect induced by the gold-coated nanotip microarrays. Such an approach introduces a reproducible method to fabricate dual spectroelectrochemical platforms allowing sequential SECM experiments and SERS sensing.

\section{Experimental section}

\subsection{Materials}

Ferrocenedimethanol, mercaptobenzoic acid (MBA, 99\%), ammonium fluoride (99.99\%), hydrofluoric acid $(99.99 \%)$ and potassium nitrate were purchased from Sigma-Aldrich. Glassy carbon electrode ( $3 \mathrm{~mm}$ diameter) were obtained from $\mathrm{CH}$ Instruments. Electrical contacts were made with high purity Silver paint (SPI, West Chester, PA, USA). The contact wires were Kynar Insulated 30 awg wrapping wires (Radiospares). The photoresist AZ1518 was obtained from AZ Electronics Materials. All aqueous solutions were prepared with Milli-Q purified water (Millipore).

\subsection{Fabrication}

Coherent optical fiber bundles with a total diameter of $350 \mu \mathrm{m}$ comprising 6000 individually cladded 3-4 $\mu$ m diameter cores (IGN-035/06) were purchased from Sumitomo Electric Industries (Japan). The insulating jacket of the bundle was removed with dichloromethane before etching, and sonicated in water for 30 s to remove any residuals. The distal face of the array was cleaved before use. The etching of the fibers was accomplished through the combination of several etching methods reported in the literature [50-52,59]. The HF solution used for etching was prepared by mixing $500 \mu \mathrm{L}$ of $40 \%$ (wt/wt) aqueous $\mathrm{NH}_{4} \mathrm{~F}$ solution and $100 \mu \mathrm{L}$ of a $48 \% \mathrm{HF}$ solution. (Caution: HF etching solutions are extremely corrosive!) The cleaved side was dipped into the $\mathrm{HF}$ etching solution and left for $3 \mathrm{~h}$. The etched bundle was then washed with water. After a drying step, it was evenly coated with a 40-nm-thick gold film with a magnetron sputtering setup (Emitech K550X). The gold-coated nanotip array was electrically connected to a copper wire with a silver paste and mechanically insulated on the side with a varnish. The deposition of this insulating varnish leads to a total diameter of the probe of around $500 \mu \mathrm{m}$. A positive photoresist AZ1518 (Microchemicals) was spin-coated on the surface of the array at $220 \mathrm{rpm}$ using a Caframo BDC-6015-200 device, which is adapted to the cylindrical geometry of the optical fiber bundles. The thickness of this insulating photoresist layer was tuned from $4 \mu \mathrm{m}$ to $10 \mu \mathrm{m}$ by changing the duration of the spin-coating step from $10 \mathrm{~s}$ to $2 \mathrm{~s}$, respectively. It was kept in the dark at room temperature for $45 \mathrm{~min}$ and then cured at $90^{\circ} \mathrm{C}$

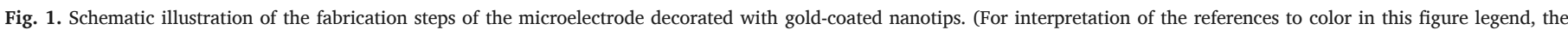
reader is referred to the Web version of this article.) 
for $20 \mathrm{~min}$. A confocal fluorescence microscope (Leica SP5, objective $20 \times$ ), was used to illuminate ( $458 \mathrm{~nm}$ wavelength, point bleach method at $10 \%$ amplitude for $3 \mathrm{~s}$ ) a region of interest on the photoresist, typically of a disk shape without any additional mask. The photoresist was then developed with a specific developer AZ726 MIF, leaving the disk pattern. The open disk diameter was tuned by adjusting the diameter of the illuminated region using the confocal mode. The sample was washed in water for $2 \mathrm{~min}$ and cured at $100^{\circ} \mathrm{C}$ for $15 \mathrm{~min}$. It caused the local removal of the irradiated photoresist area and left a gold disk microelectrode with protruding nanotips.

\subsection{Voltammetric experiments}

Cyclic voltammograms were recorded with a $\mu$-Autolab type III (Metrohm, Netherlands) potentiostat. A classical 3-electrode setup was used with the fabricated nanotip microelectrode as the working electrode, a platinum wire as a counter-electrode and a $\mathrm{Ag} / \mathrm{AgCl} / \mathrm{KCl} 3 \mathrm{M}$ reference electrode.

\subsection{Electrochemical simulations}

Simulations of the coupled diffusive and kinetic responses were modeled with COMSOL Multiphysics v5.0 in 3D geometry with the electroanalysis module. Because of the nanotips presence at the surface of the electrode, the 2D axial symmetry, usually exploited to model microelectrode arrays, was not relevant in this case. Godino et al. have shown that the $2 \mathrm{D}$ modeling is suitable only when more microelectrodes are located in the inner part than in peripheral positions [60]. With a 2D modeling, it implies that each nanotip has the same surrounding. This is true only if the number of nanotips is high. In our case where only few nanotips are present on the active surface, it is not correct to make this assumption. Therefore, the simulations were performed in 3D. To simplify the calculation, just one-quarter of the geometry was modeled (Fig. S2). The simulations are detailed in the Supporting Information. Comsol files are available upon request.

\subsection{SECM experiments}

Measurements were performed using a CHI 920C (CH Instruments, Austin, TX, USA) SECM bipotentiostat. The electrochemical cell furnished with the SECM was used to realize approach curves either on an insulating surface or on a conducting one. The insulating surface was a glass slide, in this case a typical three-electrodes configuration was used. The conducting surface was a glassy carbon electrode where a potential was applied to consume at a diffusion-limited rate the mediator generated at the tip. The reference electrode was a $\mathrm{Ag} / \mathrm{AgCl} / \mathrm{KCl} 3 \mathrm{M}$ and the counter-electrode was a platinum wire. All experiments were carried out in a Faraday cage at room temperature.

\subsection{Raman spectroscopy}

The formation of MBA self-assembled monolayer was achieved by an overnight immersion of the fabricated nanotip microelectrodes in a $0.5 \mathrm{mM}$ MBA solution in ethanol. The surface was then washed thoroughly with ethanol to remove any non-adsorbed molecules prior to characterization. SERS spectra were recorded on a Labram HR (Horiba-Jobin-Yvon) confocal microscope using an $\mathrm{Ar}-\mathrm{Kr}$ laser radiation of $647 \mathrm{~nm}$ in a backscattering configuration. The input laser beam was polarized alongside the transverse direction relative to the nanotip long axes. The laser power was $0.09 \mathrm{~mW}$. The sample was mounted on a $\mathrm{XYZ}$ piezoelectric stage positioned on the confocal microscope equipped with an Olympus short working distance $100 \times$ objective (0.9 N.A.) with the advantage of mapping the sample in $3 \mathrm{D}$ in order to focus the laser beam at the apex of a nanotip, thus enhancing the Raman signal to collect the highest intensity.

\section{Results and discussion}

\subsection{Microfabrication of the microelectrodes decorated with gold-coated nanotips}

We fabricated a dual SECM/SERS probe (Fig. 1) by adapting methodologies that derive from the preparation of classical scanning near-field optical apertures, being based on etched optical fibers [51,61-64] and photolithography protocols using photoresists. The 4-step process developed herein is schematized in Fig. 1. In brief, a nanotip array was prepared by wet chemical etching of a coherent optical fiber bundle comprising 6000 individually-cladded optical fibers of $3-4 \mu \mathrm{m}$ diameter $[50,51]$. The surface of the nanotip array was then sputter-coated with a thin gold film. Then, the entire array was insulated by spin-coating a positive photoresist on its overall surface (nanotips-decorated top surface as well as the bundle sides; step 3 in Fig. 1). The spin-coating parameters were chosen to fully cover the nanotips in height over the whole bundle, corresponding typically to $10 \mu \mathrm{m}$-thickness of photoresist. This prevents from the appearance of protruding nanotips and possibly of merging currents from different regions. The final step (Fig. 1) consisted in using a confocal microscope to illuminate a defined region, typically a micrometric disk, and form a hole in the photoresist layer. After a development step, the underlying gold-coated nanotip disk was exposed and ready to be characterized. The confocal approach was preferred to the usual 2D illuminations through a mask, which is inadequate for 3D objects, such as a cylinder-based electrode. Note that the gold film kept its original conductivity for electrochemical reactions and conferred simultaneously plasmonic properties to the sharp tips and thus to the whole nanotips-decorated microelectrodes.

Fig. 2a shows the scanning electron micrographs of microelectrode surfaces. One can observe the ordered nanotips, which are surrounded by the insulating layer defining a disk area. By adapting the region of interest illuminated in the confocal mode, the final dimension of the microelectrode was tuned. Examples shown in Fig. 2 depict disk microelectrodes which radius were estimated as: $3.5 \mu \mathrm{m}, 7 \mu \mathrm{m}, 12 \mu \mathrm{m}$ and $20 \mu \mathrm{m}$, respectively (measurements on SEM images). The dimensions of the nanotips were constant but their number varied when we modified the radius of the microelectrode, i.e. the area of the exposed gold-coated surface. The $20-\mu \mathrm{m}$ radius microelectrode (Fig. 2a) comprised $\sim 105$ nanotips whereas the smallest fabricated microelectrode of $3.5 \mu \mathrm{m}$ radius consisted of only 4 exposed nanotips (Fig. 2d and corresponding top view in Fig. S1). Microelectrodes with larger dimensions can be easily prepared as well by controlling the dimensions of the region of interest for the laser irradiation of the photoresist. Finally, it is noteworthy that a microelectrode array can also be obtained using such procedure. Indeed, we could illuminate sequentially several zones of the photoresist on the bundle, allowing the removal of well-defined regions on the same sample, after the development step. For example, Fig. 3 displays the SEM image of two microelectrodes decorated with nanotips with a similar radius of $10 \pm 1 \mu \mathrm{m}$. They were simply fabricated by illuminating in confocal microscopy two distinct zones of the same etched optical fiber bundles initially covered by the insulating photoresist. However, both microelectrodes are electrically inter-connected in that case. They cannot be individually addressed by electrochemical means and their respective currents cannot be measured separately using the aforementioned fabrication procedure. 


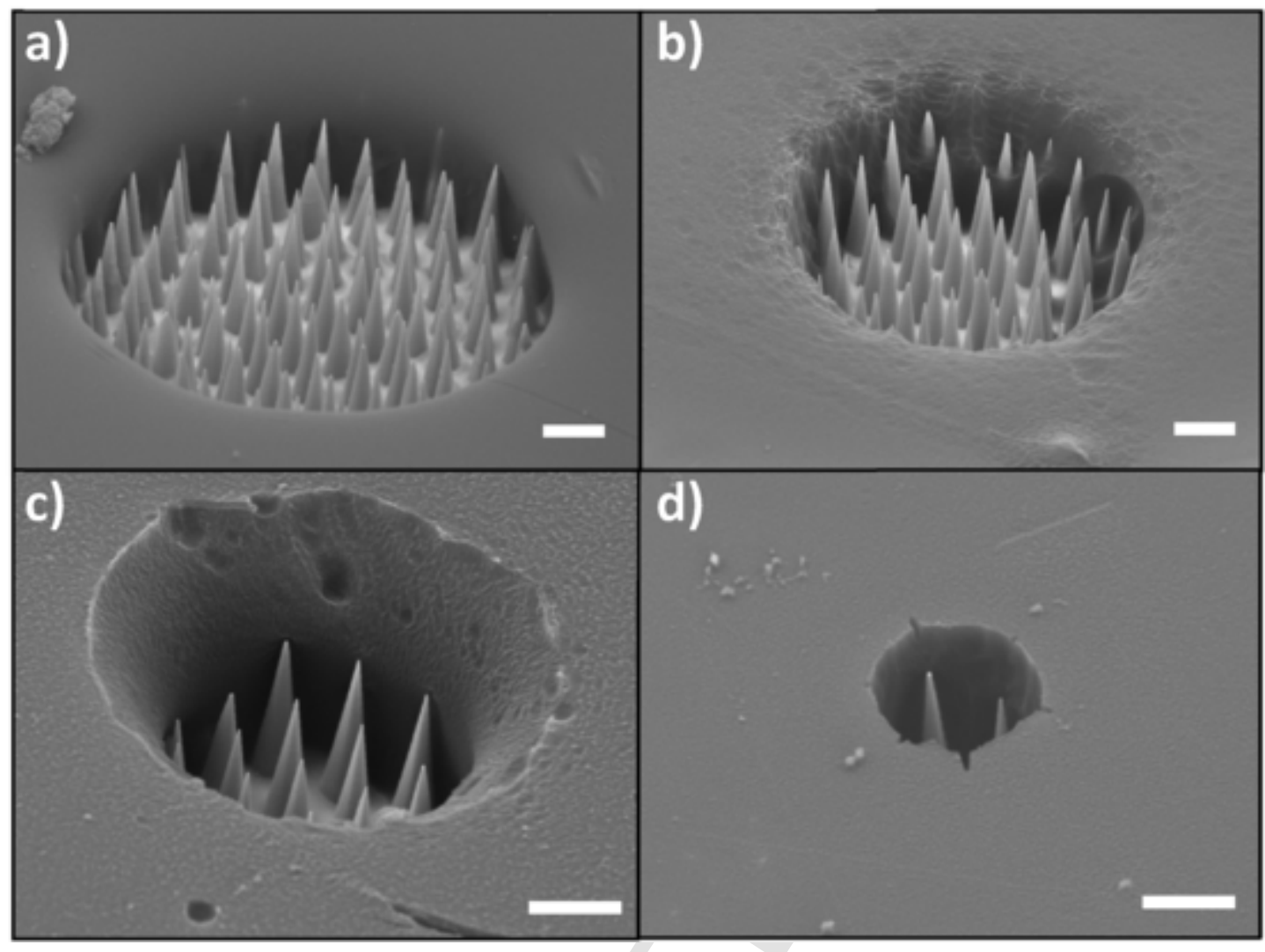

Fig. 2. SEM images of the fabricated nanotip microelectrodes with different radii: a) $20 \mu \mathrm{m}$, b) $12 \mu \mathrm{m}$, c) $7 \mu \mathrm{m}$ and d) $3.5 \mu \mathrm{m}$. White scale bars: $5 \mu \mathrm{m}$.

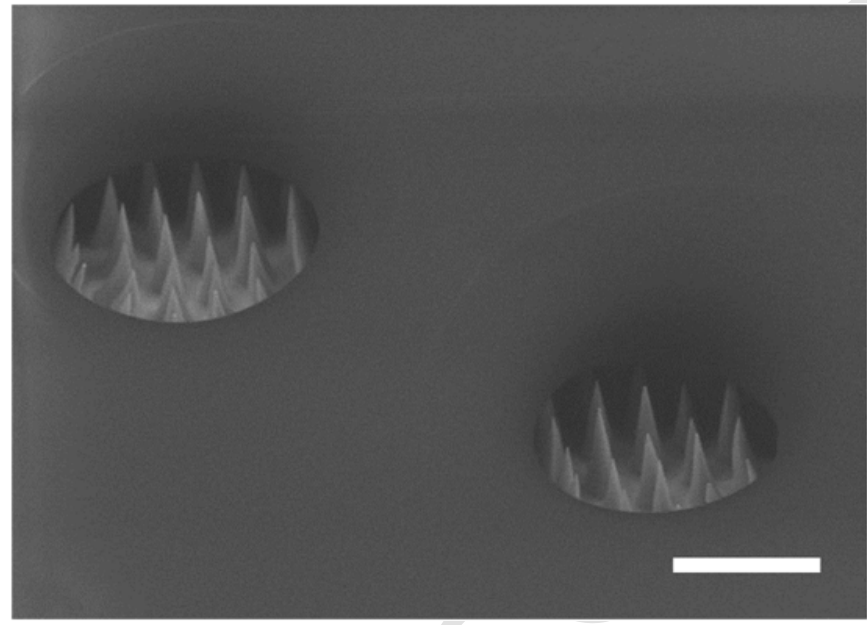

Fig. 3. SEM images of two nanotip microelectrodes fabricated by photolithography on the same gold-modified etched substrate. White scale bar: $10 \mu \mathrm{m}$.

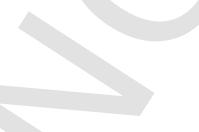

\subsection{Voltammetric characterization}

The electrochemical responses of the nanotip microelectrodes were characterized by cyclic voltammetry. Ferrocenedimethanol was selected as a redox probe because of its well-established electrochemical properties. Fig. 4 shows the voltammetric signals from the gold-coated nanotip microelectrodes shown in Fig. 2. All voltammetric responses are sigmoidal in shape indicating that nearly pure radial diffusion

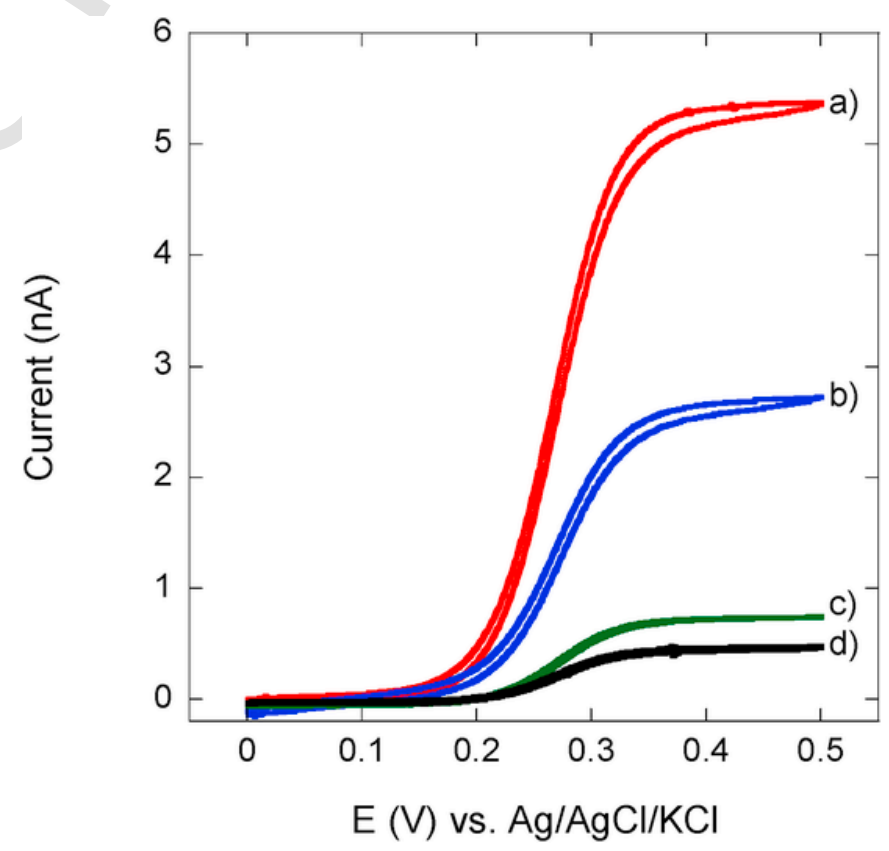

Fig. 4. Cyclic voltammograms obtained with the gold nanotip microelectrodes of different radii: a) $20 \mu \mathrm{m}$, b) $12 \mu \mathrm{m}$, c) $7 \mu \mathrm{m}$ and d) $3.5 \mu \mathrm{m}$ (SEM images in Fig. 2). The experiments were performed in an aqueous solution containing $1 \mathrm{mM}$ ferrocenedimethanol and $1 \mathrm{M} \mathrm{KNO}_{3}$. Scan rate: $2 \mathrm{mV} \mathrm{s}^{-1}$. (For interpretation of the references to color in this figure legend, the reader is referred to the Web version of this article.)

conditions were established, even with the nanotips decorating the surface of the microelectrode.

The electrochemical behavior of the nanotip microelectrodes was further investigated to understand the effects of the recessed 
geometry and of the presence of the nanotips. The radius $a$ of each microelectrode was determined from the SEM images. However, it was more difficult to measure on these the exact depth of the recess $h$, which is related to the thickness of the insulating photoresist layer. By exploiting the simulations, $h$ value was estimated (Fig. S3). We compared the simulated voltammograms with the experimental data (Fig. 4) by adjusting the value of $h$. The concentration profiles of the electrogenerated oxidized species at $\mathrm{E}=+0.5 \mathrm{~V} v$ s. $\mathrm{Ag} / \mathrm{AgCl}$ are also represented on Fig. 5a-d for the four different nanotip microelectrodes, respectively. The radius of the microelectrode, the recessed depth and the number of nanotips were chosen to correspond to the fabricated nanotip microelectrodes (Fig. 2 and Fig. S1). The concentration profiles were simulated in the 3D geometry with a microelectrode surface decorated by the nanotips. A gradient of concentrations, from $1 \mathrm{mM}$ to $0 \mathrm{mM}$ (coded as red and blue colors, respectively), is observed when moving away from the electrode surface. The shallow depth of the cavity allows the electrodes to maintain a radial diffusion layer that can clearly be observed in Fig. $5 a$ and b. In Fig. $5 c$ and d, the radial shape is retained even if it is less pronounced due to the higher depth of the cavity. Furthemore, the stationary state observed during cyclic voltammetry confirms the presence of a radial diffusion layer. A linear diffusion would give a voltammogram with a peak shape, resulting from the increase of the diffusion layer thickness over time. Due to the difference of recessed depth for the different microelectrodes, differences of calculated concentrations observed at the exit of the cavity are higher for Fig. $5 \mathrm{a}$ and $\mathrm{b}$ (concentration $\sim 0.7 \mathrm{mM}$, orange color coded) while they are lower for Fig. $5 \mathrm{c}$ and $\mathrm{d}$ conditions (concentration $\sim 0.2 \mathrm{mM}$, blue color coded). The mass transport appears linear within the cavity, but, when taking into account the depth and the low scan rate used herein, the mass transport is dominated by the radial component outside the recess. As expected, the confinement of the diffusion layer is more important when the depth of the recess microelectrode increases. This affects the value of the steady state current $i_{\text {tip }}$. The simulated voltammogram for each disk microelectrode are represented in Fig. 5e.

The difference between the classical disk flat microelectrode and the recessed microelectrode has been studied numerous times in the literature [65-67]. The deviation between the steady state currents of these two types of electrodes can be significant depending on the value of $h$. For a microdisk with a radius of $7 \mu \mathrm{m}$ and a depth of $10 \mu \mathrm{m}$, the steady state current is almost three times lower than the one obtained for a classical microelectrode with the same radius (Fig. S3). When the surface of the recessed microelectrode is additionally structured with nanotips, the steady state current increases. Indeed, the developed electroactive surface area is larger when the nanotips are present at the surface of the microelectrode. One can also observe on Fig. S3, that when the recess $h$ is $4 \mu \mathrm{m}$, for $a$ equal to 20 and $12 \mu \mathrm{m}$, the steady state current simulated for the configuration with the nanotips is very close to the one without the recess. It shows clearly that the effect of the recessed geometry affects much more the voltammetric responses than the presence of the nanotips. This result is in agreement with previous works on lithography and shear-force positioning with such nanostructured electrodes $[12,13]$.

\subsection{Investigation of the SECM behavior}

The electrochemical microscopy experiments were performed in feedback mode. In this mode the diffusion layer at the microelectrode can interact at short distances with the surface of the substrate. A sufficient anodic potential was imposed at the nanotip microelectrode to maintain a steady-state current $i_{\text {tip }}$ using ferrocenedimethanol as a reversible redox mediator. The microelectrode was moved toward the substrates at the rate of $1 \mu \mathrm{m} \mathrm{s}^{-1}$. The resulting approach curves correspond to the variations of the normalized microelectrode current, $I=i_{\text {tip }} / i_{\text {tip }, \text { inf }}$ (where $i_{\text {tip,inf }}$ is the current when the tip is positioned far away from the substrate), with the normalized distance $L=d / a$ ( $d$ is the distance between the substrates and the microelectrode).

The experimental approach curves recorded with a nanotip microelectrode have been compared (Fig. 6) to the theoretical approach curves simulated either for an insulating surface or for a conductive substrate. A glass slide was used as the insulating surface and a glassy carbon electrode as the conductive surface. On the glass slide, the current measured at the tip decreases by reducing the distance between the microelectrode and the insulator (Fig. 6). This current decreased is called a negative feedback mode. Indeed, the surface hinders the diffusion of the electroactive species that should react at the microelectrode surface. In contrast, on the glassy carbon electrode, when a potential of $0 \mathrm{~V}$ is applied to consume the species generated at the microelectrode, the current increases when the distance $L$ decreases because of the additional flux of the initial species provided by the glassy carbon surface that reach the nanotip microelectrode.

Both approach curves were realized with a nanotip microelectrode of $20 \mu \mathrm{m}$ radius, but the total diameter of the microelectrode was around $480 \mu \mathrm{m}$. Our SECM microscope is equipped with an adjustable stage for the tilt correction that allows us to achieve such
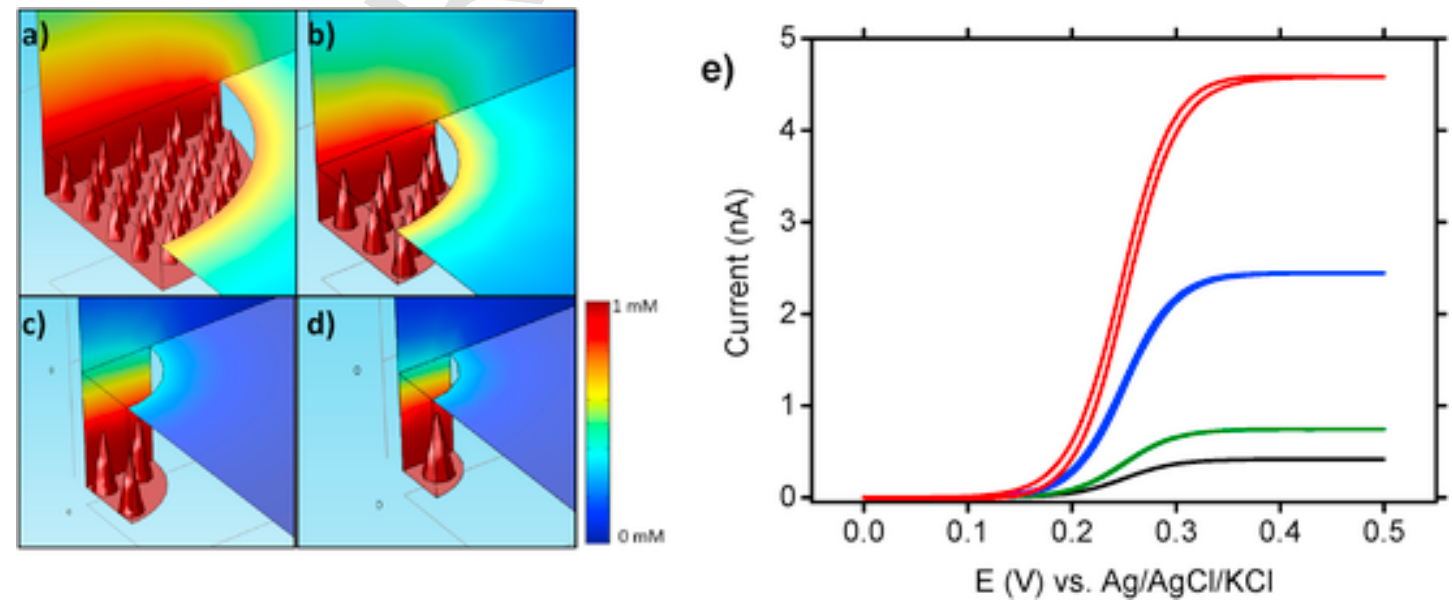

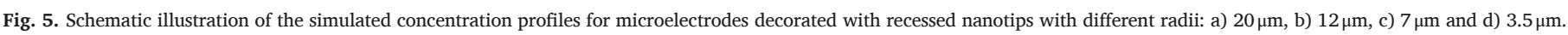

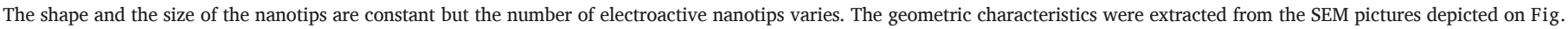

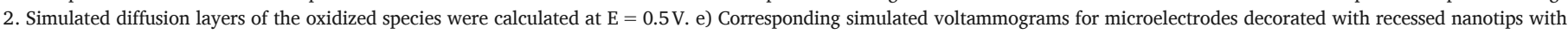

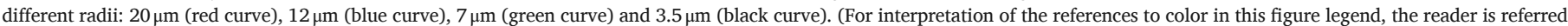
to the Web version of this article.) 


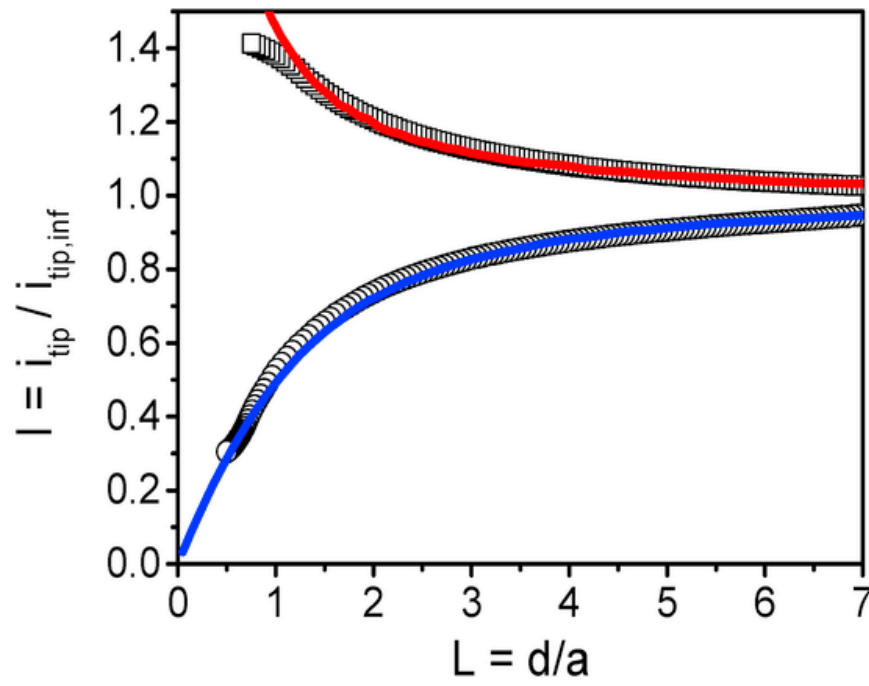

Fig. 6. SECM approach curves on $(\square)$ a glassy carbon electrode or $(O)$ a glass slide using a nanotip microelectrode of $20-\mu \mathrm{m}$ radius at a scan rate of $1 \mu \mathrm{m} \mathrm{s}^{-1}$. The tip potential was set at $0.5 \mathrm{~V}$ vs. $\mathrm{Ag} / \mathrm{AgCl} / \mathrm{KCl} 3 \mathrm{M}$. The corresponding simulated approach curves on an insulating surface (blue curve) and on a conductive surface (red curve) are superimposed. (For interpretation of the references to color in this figure legend, the reader is referred to the Web version of this article.)

approach curves. These experiments prove that these new microelectrodes are suitable for SECM experiments. Unlike to the classic disk microelectrode probe, the approach curves for both negative and positive feedback modes are not only dependent on two dimensionless parameters, $R G$ and $L, R G$ being the diameter of the surrounding insulator divided by the radius $a$ of the microelectrode. The value of $r_{g}$ is constant $(250 \mu \mathrm{m})$ but, since we tuned the radius of the microelectrode in this work, the normalized parameter RG varies. In our simulations, we had to take into account the depth of the recessed microelectrode and the shape of the nanotips. These two geometrical features made more complex the modeling of currents but do not prevent from obtaining simulated approach curves. These experiments prove that these new microelectrodes are suitable for SECM experiments. Furthermore the experimental curves are in good agreement with the simulated curves (Fig. 6). This study demonstrates that these new nanotips-decorated microelectrodes can be used in an SECM configuration with the support of the simulations to analyze the experimental data.

Even if we successfully perform such SECM experiments, considering the large diameter of the probe, it can be difficult to approach it very close to a surface or to make in vivo studies using it. On the one hand, in previous reports, we used probes of similar sizes in SECM mode to pattern a surface or combined with shear-force positioning [12,13]. On the other hand, to make easier such in vivo experiments, it would be possible to decrease the global size of the bundle by heating-pulling techniques. As an example, a pulled and etched optical fiber bundle with a final radius of $22 \mu \mathrm{m}$ is displayed in Fig. S4. It shows also that the aspect ratio of the nanotips is conserved.

\subsection{SERS experiments}

The capability to detect SERS signals with the developed nanotip microarrays was also investigated. Etched optical fiber bundles exhibiting a larger diameter $(300 \mu \mathrm{m})$ have already shown promising capabilities as SERS-active substrates $[47,48]$. However, the presence of the surrounding photoresist film required to define a disk microelectrode could potentially affect the plasmonic behavior and lower the SERS efficiency. MBA was chosen as a model compound to record the Raman spectroscopic features because it is known to form stable monolayers on gold or silver metal surfaces $[68,69]$. Therefore, the thin gold layer deposited on the microarray was modified with a MBA monolayer by performing an overnight immersion and self-assembly with a $0.5 \mathrm{mM}$ MBA solution in ethanol. Fig. 7a shows a typical SERS
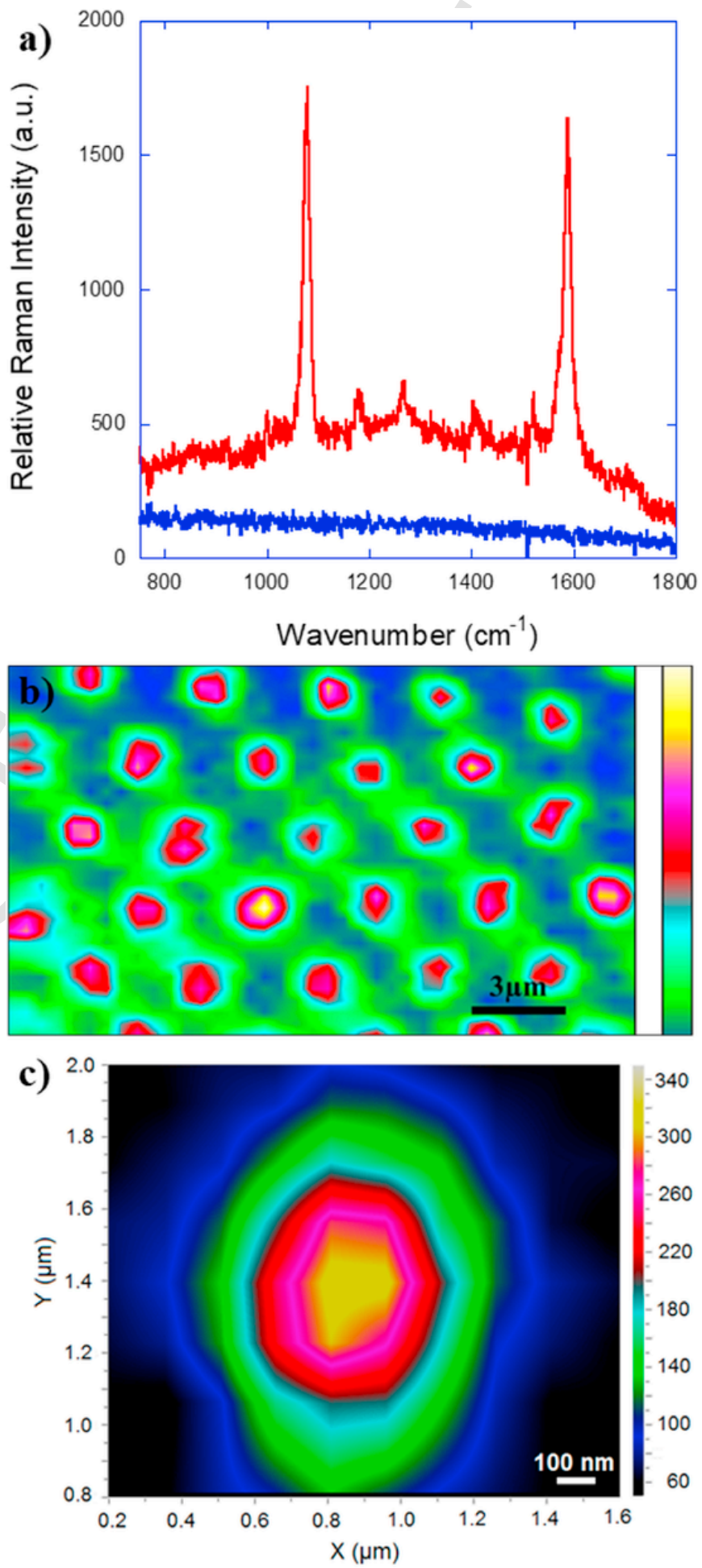

Fig. 7. a) Typical SERS spectra of MBA recorded on the gold-coated nanotip microelec trode (red line) and negative control on a non-structured zone (blue line). b) Confocal Raman mapping of the microelectrode functionalized with a MBA monolayer. The images are color-coded according to the intensity of the Raman band of MBA adsorbed species centred at $1591 \mathrm{~cm}^{-1}$. c) Confocal Raman image $(1.4 \mu \mathrm{m} \times 1.2 \mu \mathrm{m})$ of an individual gold-coated nanotip modified with a MBA self-assembled monolayer. (For interpretation of the references to color in this figure legend, the reader is referred to the Web version of this article.) 
spectrum recorded on a functionalized-nanotip microelectrode $(647 \mathrm{~nm}$, $0.09 \mathrm{~mW}, 100 \times$ objective, N.A. 0.9). These data match perfectly with MBA characteristic spectrum, which displays two main features appearing as intense bands at 1078 and $1591 \mathrm{~cm}^{-1}$ and being assigned to $\nu_{8 \mathrm{a}}$ and $\nu_{12}$ aromatic ring vibrations, respectively $[68,69]$. For comparison, a Raman spectrum was also recorded from a MBA solution in pure ethanol $(647 \mathrm{~nm}, 10 \mathrm{~mW}, 20 \times$ objective, N.A. 0.35 , data not shown) and this spectrum was consistent with literature [68] showing the same two bands centred at $1099 \mathrm{~cm}^{-1}$ and $1600 \mathrm{~cm}^{-1}$. It is noteworthy that the relative intensities of the vibration modes of the MBA molecules adsorbed on the nanostructured gold surface are indeed slightly different from that of the reference spectrum. The Raman enhancement factor, which directly originates from electromagnetic enhancement processes at the tip apex was calculated according to a previously reported method [48], and a typical value of $7 \pm 1 \times 10^{4}$ was found (see SI for details). The standard deviation originates from the tip-to-tip sharpness difference based on a series of spectra recorded independently on 4 nanotips, thus modulating the intrinsic SERS efficiency. One can also observe that the background of the spectrum is significant though not limiting analytically, depicting a typical large broad feature assigned to fluorescence and superimposed to the sharp Raman peaks. This background was not only observed on the nanotips (at the center of the disk microarray) but also on its border where the photoresist layer was not removed (Fig. 7b). We did not investigate in detail the nature of such a fluorescence background as Raman signal could clearly be discriminated but it could be possibly due to some residual constituents of the photoresist layer. This potential issue could be solved by using some other insulating materials such as fluorocarbon polymer thin films as demonstrated for the controlled positioning of analytes and cells on a plasmonic platform [70].

In addition to the recording of Raman spectra, high-resolution spatial-imaging measurements were also performed in order to study the influence of the XYZ position on the SERS substrates. The mapping of a series of nanotips was performed by recording step spectra every $150 \mathrm{~nm}$ with an integration time of $3 \mathrm{~s}$. Fig. 7c shows the Raman intensity distribution obtained by selecting the MBA typical band at $1591 \mathrm{~cm}^{-1}$. This is indeed a representative image of a single tip optimized by varying the axial position $(\mathrm{Z})$ and recorded on a $1.4 \mu \mathrm{m} \times 1.2 \mu \mathrm{m} 2 \mathrm{D}$ zone $(\mathrm{XY})$. This data confirms that the Raman enhancement can be finely tuned alongside the tip with the maximum intensity perfectly matching the summit of each tip structure.

Moreover, in the optical configurations reported so far where SECM and Raman (or SERS) spectroscopy have been combined, the electrochemical probes have to be manipulated and positioned under a Raman microscope $[20,29,71]$. It represents an experimental challenge to associate both microscopies in a single setup. With the dual probes proposed herein, the optical configuration for the SECM/SERS experiments may be different. Indeed, the developed probes are based on optical fiber bundles that guide light. In a previous report [47], we demonstrated the remote SERS sensing through such nanostructured bundles. The excitation laser was injected in the polished proximal face of the optical bundle. It was guided by total internal reflection in the individual fiber cores in order to be confined to the apex of the tips and induce a SERS effect. A fraction of the backscattered Raman signal at the tips is then collected by the same bundle, transmitted and detected back at the proximal face of the bundle. This optical configuration associated with the reported dual probes allows both Raman and SECM setups to be separated. Indeed, the Raman setup can operate at the proximal extremity of the bundle whereas SECM setup is located at the distal extremity.

Besides, the recessed geometry of the reported dual probes does not allow a priori to position them in the near-field of a given substrate or surface. It means that TERS or SERS imaging experiments of immobilized molecules of interests at a living cell surface, for example, are not possible in this configuration. Indeed, the tips would be located too far from the surface to benefit from the plasmonic near field effects. However, this probe can detect freely-diffusing molecules by SERS. Molecules that are released by cells $[72,73]$ or (eletro)generated by a surface $[74,75]$ diffuse and reach the nanotips where they are detected by SERS and/or by electrochemistry. In addition, it renders possible the study of the molecular functionalization and substitution dynamics of surfaces as well as the characterization of cellular release of biomolecules $[36,76]$. In these cases, SECM mode can be used to position the probe or to examine surface reactivity while SERS sensing is devoted to the highly specific detection of free molecules in the surrounding liquid medium.

\section{Conclusion}

We prepared a new type of dual SECM/SERS platforms by combining the fabrication processes of scanning near-field optical probes with photolithography. The resulting gold-coated microelectrodes decorated with optical nanotips were fabricated with tunable size. The sigmoidal shape of the steady-state voltammograms indicates that these opto-electrochemical probes behave as microelectrodes with radial diffusion regime. Numerical simulations show that the recessed geometry governs mainly the voltammetric behavior whereas the presence of the nanotips influences slightly the diffusional regime and the electrochemical responses. Such nanostructured microelectrodes are then used as SECM microprobes in negative and positive feedback modes on insulating and conducting substrates, respectively. Finally, a SERS effect induced by the gold-coated nanotips was demonstrated by imaging a monolayer of a chemical probe adsorbed on the microelectrode surface by confocal Raman microscopy. Such dual platforms show distinct and complementary features for investigating complex microenvironments or in situ SERS studies of electrochemically induced processes. They could find a wide range of applications in photonic devices or electro-optical probes for biosensing, among which the detection of neurosecretory processes at single cells. Indeed, as shown recently [73], SERS performed at a nanotip allows one to detect dynamically the cell release of certain neurotransmitters which are not electroactive (glutamate, ATP), others being electroactive (dopamine) could be detectable by the electrode surface simultaneously. The combination of both methodologies on the same probe, of less than $100 \mu \mathrm{m}$ diameter, would provide unprecedented features for sensing, for instance, in the brain tissue different types of neuronal activities with high spatial accuracy.

\section{Acknowledgements}

We thank the Agence Nationale de la Recherche (Moly project, ANR-15-CE19-0005-02), University of Bordeaux, Bordeaux INP and CNRS for their financial supports.

\section{Appendix A. Supplementary data}

Supplementary data to this article can be found online at https://doi. org/10.1016/j.electacta.2019.135105.

\section{References}

[1] A.J. Bard, M.V. Mirkin, Scanning Electrochemical Microscopy, second ed., CRC Press, 2012.

[2] M.V. Mirkin, F.-R.F. Fan, A.J. Bard, Scanning electrochemical microscopy part 13. Evaluation of the tip shapes of nanometer size microelectrodes, J. Electroanal. Chem. 328 (1992) 47.

[3] Q. Fulian, A.C. Fisher, G. Denuault, Applications of the boundary element method in Electrochemistry: scanning electrochemical microscopy, J. Phys. Chem. B 103 (1999) 4387-4392.

[4] Q. Fulian, A.C. Fisher, G. Denuault, Applications of the boundary element method in Electrochemistry: scanning electrochemical microscopy, Part 2, J. Phys. Chem. B 103 (1999) 4393-4398. 
[5] Y. Selzer, D. Mandler, Scanning electrochemical microscopy. Theory of the feedback mode for hemispherical Ultramicroelectrodes: steady-state and transient behavior, Anal. Chem. 72 (2000) 2383-2390.

[6] C. Amatore, B. Fosset, Equivalence between microelectrodes of different shapes: between myth and reality, Anal. Chem. 68 (1996) 4377-4388.

[7] H. Xiong, D.A. Gross, J. Guo, S. Amemiya, Local feedback mode of scanning electrochemical microscopy for electrochemical characterization of one-dimensional Nanostructure: theory and experiment with nanoband electrode as model substrate, Anal. Chem. 78 (2006) 1946-1957.

[8] Y. Lee, S. Amemiya, A.J. Bard, Scanning electrochemical microscopy. 41. Theory and characterization of ring electrodes, Anal. Chem. 73 (2001) 2261-2267.

[9] E.M. Hussien, W. Schuhmann, A. Schulte, Shearforce-based constant-distance scanning electrochemical microscopy as fabrication tool for needle-type carbon-fiber nanoelectrodes, Anal. Chem. 82 (2010) 5900-5905.

[10] P. Sun, M.V. Mirkin, Scanning electrochemical microscopy with slightly recessed nanotips, Anal. Chem. 79 (2007) 5809-5816.

[11] A. Schulte, M. Nebel, W. Schuhmann, Scanning electrochemical microscopy in neuroscience, Annu. Rev. Anal. Chem. 3 (2010) 299-318.

[12] C. Adam, F. Kanoufi, N. Sojic, M. Etienne, Shearforce positioning of nanoprobe electrode arrays for scanning electrochemical microscopy experiments, Electrochim. Acta 179 (2015) 45-56.

[13] F. Deiss, C. Combellas, C. Fretigny, N. Sojic, F. Kanoufi, Lithography by scanning electrochemical microscopy with a multiscaled electrode, Anal. Chem. 82 (2010) 5169.

[14] F. Cortés-Salazar, D. Momotenko, H.H. Girault, A. Lesch, G. Wittstock, Seeing big with scanning electrochemical microscopy, Anal. Chem. 83 (2011) 1493-1499.

[15] J. Wiedemair, B. Balu, J.-S. Moon, D.W. Hess, B. Mizaikoff, C. Kranz, Plasma-deposited fluorocarbon films: insulation material for microelectrodes and combined atomic force Microscopy-Scanning electrochemical microscopy probes, Anal. Chem. 80 (2008) 5260-5265.

[16] P. Knittel, B. Mizaikoff, C. Kranz, Simultaneous nanomechanical and electrochemical mapping: combining peak force tapping atomic force microscopy with scanning electrochemical microscopy, Anal. Chem. 88 (2016) 6174-6178.

[17] A. Lesch, B. Vaske, F. Meiners, D. Momotenko, F. Cortés-Salazar, H.H. Girault, G. Wittstock, Parallel imaging and template-free patterning of self-assembled monolayers with soft linear microelectrode arrays, Angew. Chem. Int. Ed. 51 (2012) 10413-10416.

[18] Y. Lee, A.J. Bard, Fabrication and characterization of probes for combined scanning electrochemical/optical microscopy experiments, Anal. Chem. 74 (2002) 3626-3633.

[19] R. Lei, L. Stratmann, D. Schäfer, T. Erichsen, S. Neugebauer, N. Li, W. Schuhmann, Imaging biocatalytic activity of enzyme-polymer spots by means of combined scanning electrochemical microscopy/electrogenerated chemiluminescence, Anal. Chem. 81 (2009) 5070.

[20] M. Etienne, M. Dossot, J. Grausem, G. Herzog, Combined Raman microspectrometer and shearforce regulated SECM for corrosion and self-healing analysis, Anal. Chem. 86 (2014) 11203-11210.

[21] L. Wang, J. Kowalik, B. Mizaikoff, C. Kranz, Combining scanning electrochemical microscopy with infrared attenuated total reflection spectroscopy for in situ studies of electrochemically induced processes, Anal. Chem. 82 (2010) 3139-3145.

[22] L. Wang, C. Kranz, B. Mizaikoff, Monitoring scanning electrochemical microscopy approach curves with mid-infrared spectroscopy: toward a novel current-independent positioning mode, Anal. Chem. 82 (2010) 3132-3138.

[23] K. Maruyama, H. Ohkawa, S. Ogawa, A. Ueda, O. Niwa, K. Suzuki, Fabrication and characterization of a nanometer-sized optical fiber electrode based on selective chemical etching for scanning electrochemical/optical microscopy, Anal. Chem. 78 (2006) 1904-1912.

[24] F.-M. Boldt, J. Heinze, M. Diez, J. Petersen, M. Börsch, Real-time pH microscopy down to the molecular level by combined scanning electrochemical microscopy/single-molecule fluorescence spectroscopy, Anal. Chem. 76 (2004) 3473-3481.

[25] S. Szunerits, N. Knorr, R. Calemczuk, T. Livache, New approach to writing and simultaneous reading of Micropatterns: combining surface plasmon resonance imaging with scanning electrochemical microscopy (SECM), Langmuir 20 (2004) 9236-9241.
[26] G. Premaratne, J. Niroula, M.K. Patel, W. Zhong, S.L. Suib, A.K. Kalkan, S. Krishnan, Electrochemical and surface-plasmon correlation of a serum-autoantibody immunoassay with binding insights: graphenyl surface versus mercapto-monolayer surface, Anal. Chem. 90 (2018) 12456-12463.

[27] H. He, Z. Ding, D.W. Shoesmith, The determination of electrochemical reactivity and sustainability on individual hyper-stoichiometric UO2 $+\mathrm{x}$ grains by Raman microspectroscopy and scanning electrochemical microscopy, Electrochem. Commun. 11 (2009) 1724-1727.

[28] L. Guadagnini, C. Chiavari, C. Martini, E. Bernardi, L. Morselli, D. Tonelli, The use of scanning electrochemical microscopy for the characterisation of patinas on copper alloys, Electrochim. Acta 56 (2011) 6598-6606.

[29] J. Clausmeyer, M. Nebel, S. Grützke, Y.U. Kayran, W. Schuhmann, Local surface modifications investigated by combining scanning electrochemical microscopy and surface-enhanced Raman scattering, ChemPlusChem 83 (2018) 414-417.

[30] M.E. Stewart, C.R. Anderton, L.B. Thompson, J. Maria, S.K. Gray, J.A. Rogers, R.G. Nuzzo, Nanostructured plasmonic sensors, Chem. Rev. 108 (2008) 494-521.

[31] S. Cintra, M.E. Abdelsalam, P.N. Bartlett, J.J. Baumberg, T.A. Kelf, Y. Sugawara, A.E. Russell, Sculpted substrates for SERS, Faraday Discuss 132 (2006) 191.

[32] M.E. Abdelsalam, S. Mahajan, P.N. Bartlett, J.J. Baumberg, A.E. Russell, SERS at structured palladium and platinum surfaces, J. Am. Chem. Soc. 129 (2007) 7399-7406.

[33] J.N. Anker, W.P. Hall, O. Lyandres, N.C. Shah, J. Zhao, R.P. Van Duyne, Biosensing with plasmonic nanosensors, Nat. Mater. 7 (2008) 442-453.

[34] K.A. Willets, R.P. Van Duyne, Localized surface plasmon resonance spectroscopy and sensing, Annu. Rev. Phys. Chem. 58 (2006) 267-297.

[35] D. Kurouski, M. Mattei, R.P. Van Duyne, Probing redox reactions at the nanoscale with electrochemical tip-enhanced Raman spectroscopy, Nano Lett. 15 (2015) 7956-7962.

[36] S. Zaleski, A.J. Wilson, M. Mattei, X. Chen, G. Goubert, M.F. Cardinal, K.A. Willets, R.P. Van Duyne, Investigating nanoscale electrochemistry with surfaceand tip-enhanced Raman spectroscopy, Acc. Chem. Res. 49 (2016) 2023-2030.

[37] M. Fan, G.F.S. Andrade, A.G. Brolo, A review on the fabrication of substrates for surface enhanced Raman spectroscopy and their applications in analytical chemistry, Anal. Chim. Acta 693 (2011) 7-25.

[38] R.J.C. Brown, M.J.T. Milton, Nanostructures and nanostructured substrates for surface - enhanced Raman scattering (SERS), J. Raman Spectrosc. 39 (2008) 1313-1326.

[39] L. Zhao, J. Blackburn, C.L. Brosseau, Quantitative detection of uric acid by electrochemical-surface enhanced Raman spectroscopy using a multilayered $\mathrm{Au} / \mathrm{Ag}$ substrate, Anal. Chem. 87 (2015) 441-447.

[40] M. Atighilorestani, J.W. Menezes, A.G. Brolo, Large area plasmonic gold nanopillar 3-D electrodes, Electrochim. Acta 188 (2016) 91-97.

[41] F. Deiss, N. Sojic, D. White, P. Stoddart, Nanostructured optical fibre arrays for high-density biochemical sensing and remote imaging, Anal. Bioanal. Chem. 396 (2010) 53.

[42] G. Kostovski, D.J. White, A. Mitchell, M.W. Austin, P.R. Stoddart, Nanoimprinted optical fibres: biotemplated nanostructures for SERS sensing, Biosens. Bioelectron. 24 (2009) 1531.

[43] D.J. White, A.P. Mazzolini, P.R. Stoddart, Fabrication of a range of SERS substrates on nanostructured multicore optical fibres, J. Raman Spectrosc. 38 (2007) 377-382.

[44] D.J. White, P.R. Stoddart, Nanostructured optical fiber with surface-enhanced Raman scattering functionality, Opt. Lett. 30 (2005) 598-600.

[45] M. Fan, P. Wang, C. Escobedo, D. Sinton, A.G. Brolo, Surface-enhanced Raman scattering (SERS) optrodes for multiplexed on-chip sensing of nile blue A and oxazine 720, Lab Chip 12 (2012) 1554-1560.

[46] G.F.S. Andrade, M. Fan, A.G. Brolo, Multilayer silver nanoparticles-modified optical fiber tip for high performance SERS remote sensing, Biosens. Bioelectron. 25 (2010) 2270.

[47] V. Guieu, P. Garrigue, F. Lagugné-Labarthet, L. Servant, N. Sojic, D. Talaga, Remote surface enhanced Raman spectroscopy imaging via a nanostructured optical fiber bundle, Opt. Express 17 (2009) 24030.

[48] V. Guieu, D. Talaga, L. Servant, N. Sojic, F. Lagugne-Labarthet, Multitip-localized enhanced Raman scattering from a nanostructured optical fiber array, J. Phys. Chem. C 113 (2009) 874-881. 
[49] V. Guieu, F. Lagugné-Labarthet, L. Servant, D. Talaga, N. Sojic, Ultrasharp optical-fiber nanoprobe array for Raman local-enhancement imaging, Small 4 (2008) 96-99.

[50] P. Pantano, D.R. Walt, Ordered nanowell Arrays, Chem. Mater. 8 (1996) 2832-2835.

[51] P. Pantano, D.R. Walt, Toward a near-field optical array, Rev. Sci. Instrum. 68 (1997) 1357-1359.

[52] P. Pantano, D.R. Walt, Analytical applications of optical imaging fibers, Anal. Chem. 67 (1995) 481A-487A

[53] M. Zamuner, D. Talaga, V. Guieu, F. Deiss, A. Kuhn, P. Ugo, N. Sojic, Multiscaled nanostructured array fabricated on an etched imaging bundle for surface-enhanced Raman scattering, Adv. Funct. Mater. 19 (2009) 3129-3135.

[54] H. Aouani, F. Deiss, J. Wenger, P. Ferrand, N. Sojic, H. Rigneault, Optical-fiber-microsphere for remote fluorescence correlation spectroscopy, Opt. Express 17 (2009) 19085.

[55] S. Szunerits, J.M. Tam, L. Thouin, C. Amatore, D.R. Walt, Spatially resolved electrohemiluminescence on an array of electrode tips, Anal. Chem. 75 (2003) 4382-4388.

[56] A. Chovin, P. Garrigue, N. Sojic, Remote NADH imaging through an ordered array of electrochemiluminescent nanoapertures, Bioelectrochem. 69 (2006) 25-33.

[57] A. Chovin, P. Garrigue, P. Vinatier, N. Sojic, Development of an ordered array of optoelectrochemical individually readable sensors with submicrometer dimensions: application to remote electrochemiluminescence imaging, Anal. Chem. 76 (2004) 357-364.

[58] A. Chovin, P. Garrigue, N. Sojic, Electrochemiluminescent detection of hydrogen peroxide with an imaging sensor array, Electrochim. Acta 49 (2004) 3751-3757.

[59] A. Chovin, P. Garrigue, I. Manek-Hönninger, N. Sojic, Fabrication, characterization and far-field optical properties of an ordered array of nanoapertures, Nano Lett. 4 (2004) 1965-1968.

[60] N. Godino, X. Borrisé, F.X. Muñoz, F.J. del Campo, R.G. Compton, Mass transport to nanoelectrode arrays and limitations of the diffusion domain approach: theory and experiment, J. Phys. Chem. C 113 (2009) 11119-11125.

[61] M. Ohtsu, Progress of high-resolution photon scanning tunneling microscopy due to a nanometric fiber probe, J. Light. Technol. 13 (1995) 1200-1221.

[62] G. Krausch, S. Wegscheider, A. Kirsch, H. Bielefeldt, J.C. Meiners, J. Mlynek, Near field microscopy and lithography with uncoated fiber tips: a comparison, Opt. Commun. 119 (1995) 283-288.

[63] M. De Serio, R. Zenobi, V. Deckert, Looking at the nanoscale: scanning near-field optical microscopy, Trends Anal. Chem. 22 (2003).

[64] R. Micheletto, N. Yoshimatsu, S. Okazaki, A simple method for the fabrication of low cost scanning near field optical microscope probes with acrylic paint as coating element, Opt. Commun. 188 (2001) 11-15.
[65] P.N. Bartlett, S.L. Taylor, An accurate microdisc simulation model for recessed microdisc electrodes, J. Electroanal. Chem. 453 (1998) 49-60.

[66] R. Ferrigno, P.F. Brevet, H.H. Girault, Finite element simulation of the chronoamperometric response of recessed and protruding microdisc electrodes, Electrochim. Acta 42 (1997) 1895-1903.

[67] A.M. Bond, D. Luscombe, K.B. Oldham, C.G. Zoski, A comparison of the chronoamperometric response at inlaid and recessed disc microelectrodes, $\mathrm{J}$. Electroanal. Chem. 249 (1988) 1-14.

[68] A. Michota, J. Bukowska, Surface-enhanced Raman scattering (SERS) of 4-mercaptobenzoic acid on silver and gold substrates, J. Raman Spectrosc. 34 (2003) 21-25.

[69] H. Park, S.B. Lee, K. Kim, M.S. Kim, Surface-enhanced Raman scattering of p-aminobenzoic acid at silver electrode, J. Phys. Chem. 94 (1990) 7576-7580.

[70] M. Tabatabaei, G.Q. Wallace, F.A. Caetano, E.R. Gillies, S.S.G. Ferguson, F. Lagugne-Labarthet, Controlled positioning of analytes and cells on a plasmonic platform for glycan sensing using surface enhanced Raman spectroscopy, Chem. Sci. 7 (2016) 575-582.

[71] M. Steimecke, G. Seiffarth, M. Bron, In situ characterization of $\mathrm{Ni}$ and $\mathrm{Ni} / \mathrm{Fe}$ thin film electrodes for oxygen evolution in alkaline media by a Raman-coupled scanning electrochemical microscope setup, Anal. Chem. 89 (2017) 10679-10686.

[72] R.J. Dijkstra, W.J.J.M. Scheenen, N. Dam, E.W. Roubos, J.J. ter Meulen, Monitoring neurotransmitter release using surface-enhanced Raman spectroscopy, J. Neurosci. Methods 159 (2007) 43-50.

[73] F. Lussier, T. Brulé, M.-J. Bourque, C. Ducrot, L.-É. Trudeau, J.-F. Masson, Dynamic SERS nanosensor for neurotransmitter sensing near neurons, Faraday Discuss 205 (2017) 387-407.

[74] C. Amatore, F. Bonhomme, J.-L. Bruneel, L. Servant, L. Thouin, Mapping concentration profiles within the diffusion layer of an electrode Part I. Confocal resonance Raman microscopy, Electrochem. Commun. 2 (2000) 235-239.

[75] C. Amatore, F. Bonhomme, J.-L. Bruneel, L. Servant, L. Thouin, Mapping dynamic concentration profiles with micrometric resolution near an active microscopic surface by confocal resonance Raman microscopy. Application to diffusion near ultramicroelectrodes: first direct evidence for a conproportionation reaction, J. Electroanal. Chem. 484 (2000) 1-17.

[76] M. Plissonneau, A. Madeira, D. Talaga, S. Bonhommeau, L. Servant, R.A.L. Vallée, C. Labrugère, I.A. Goldthorpe, L. Pautrot-D’Alencon, T. Le Mercier, M. Treguer-Delapierre, Efficient passivation of Ag nanowires with 11-mercaptoundecanoic acid probed using in situ total-internal-reflection surface-enhanced Raman scattering spectroscopy, ChemNanoMat 5 (2019) 1044-1049. 